\title{
Esirie
}

OPINION

\section{A story of success: continuous quality improvement in cystic fibrosis care in the USA}

\author{
Bradley S Quon, Christopher H Goss
}

\section{See Editorial, p 1020}

Division of Pulmonary and Critical Care Medicine, Department of Medicine, University of Washington Medical Center, Seattle, Washington, USA

\section{Correspondence to} Christopher H Goss, Associate Professor of Medicine University of Washington Medical Center, Campus Box 356522, 1959 N.E. Pacific Seattle, WA 98195, USA; goss@u.washington.edu

Received 12 June 2011 Accepted 28 June 2011 Published Online First 3 August 2011

\begin{abstract}
Background Continuous quality improvement (COI) in healthcare can be described as a reiterative approach to improving processes to reduce unexpected variation in health outcomes. COl represents one model to achieve quality improvement (Ql) and has long been recognized as a key to success in the manufacturing industry with companies like Toyota leading the way.

Objective Healthcare, and specifically pulmonary, critical care and sleep medicine represent ideal settings for the application of COI.
\end{abstract}

Methods This opinion piece will describe $\mathrm{Ol}$ and COl initiatives in the US Cystic fibrosis (CF) population.

Results Ol in CF care in the United States has been ongoing since inception of the US CF Foundation (CFF) in 1955. This effort has included work to improve the quality of clinical care provided at CF centers and work to improve clinical outcomes in CF. More recently, $\mathrm{Ol}$ methods have been applied to the conduct of clinical research.

Conclusions The CF community has become a leader in the area of $\mathrm{Ol}$ and has pointed out the opportunities for others to follow in the area of lung diseases.

Quality improvement (OI) in healthcare has been defined as 'an interdisciplinary process to raise the likelihood of the delivery of best practices for preventive, diagnostic, therapeutic, and rehabilitative care to maintain, restore, or improve health outcomes of individuals and populations'. ${ }^{1}$ Continuous quality improvement (COI) in healthcare can be described as a reiterative approach to improving processes to reduce unexpected variation in health outcomes. COI represents one model to achieve OI and has long been recognised as a key to success in the manufacturing industry with companies like Toyota leading the way. The role of COI has been less well adopted in the healthcare profession. ${ }^{2}$ Healthcare and specifically pulmonary, critical care and sleep medicine represent ideal settings for the application of COI.

Cystic fibrosis (CF) is an example of a pulmonary disease that has led the way in COI. OI in CF care in the USA has been ongoing since inception of the US CF Foundation (CFF) in 1955. The US CFF was founded by a coalition of parents of individuals with CF with an overarching goal of curing the disease and improving quality of life. The US CFF has invested heavily in developing a network of CF specialty care centres across the USA with the goal of improving quality of care in important areas such as nutrition, lung health and infection control. As a result of these efforts, the quality of care for people with CF has improved significantly over the past five decades and this has been associated with improved survival (the median age of predicted survival increased from 27 years in 1989 to 36 years in 2009). ${ }^{3}$ Development of the CFF Patient Registry and the CFF Therapeutic Development Network (TDN) have been the engines behind this success. ${ }^{4}$

\section{CFF PATIENT REGISTRY: REDUCING GAPS IN CLINICAL PRACTICE}

In the words of management guru Peter Drucker, 'if you can't measure it, you can't manage it.' The US CFF recognised the critical role of data collection and measurement in OI early on and thus created a patient registry in $1966 .{ }^{5}$ The registry data have evolved over the years from a few variables on vital status to allow for a better understanding of the natural history of disease to a comprehensive database that gives healthcare providers and researchers the information they need to develop care guidelines, improve delivery of care and identify eligible patients for clinical trials. The US CFF Patient Registry now includes detailed annual and encounter-based data on over 300 unique variables running the gamut from active pulmonary therapies to sputum microbiology to pulmonary function test results to CF-related comorbidities for each of its more than 26000 participants. ${ }^{3}$

Box 1 Tools and methods to achieve quality improvement $^{6}{ }^{7}$

Delineate healthcare process

- Collect data over time to document variation in care practices and clinical outcomes

- Document unwanted and unnecessary variation

- Collect information regarding customer/beneficiary knowledge (ie, measurements of illness burden, functional status, quality of life, recipients' assessment of the quality of their care)

- Adopt widespread public sharing of information

- Improve communication by building teams and enhancing group learning using specific skills (ie, situation, background, assessment and request (SBAR))

- Create a leadership plan acknowledging: leading following and making changes in healthcare

- Build knowledge (locally useful) and then take initiative and use adaptive action, reviewing and reflecting

- Make small tests of change (ie, plan-dostudy-act (PDSA) cycles) 
Median FEV \% predicted, age 6-13 years

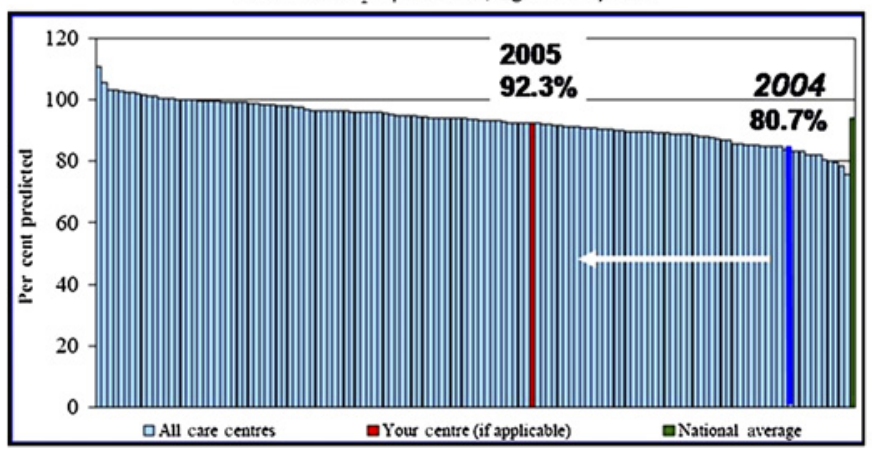

Median FEV ${ }_{1} \%$ predicted, age $13-18$ years

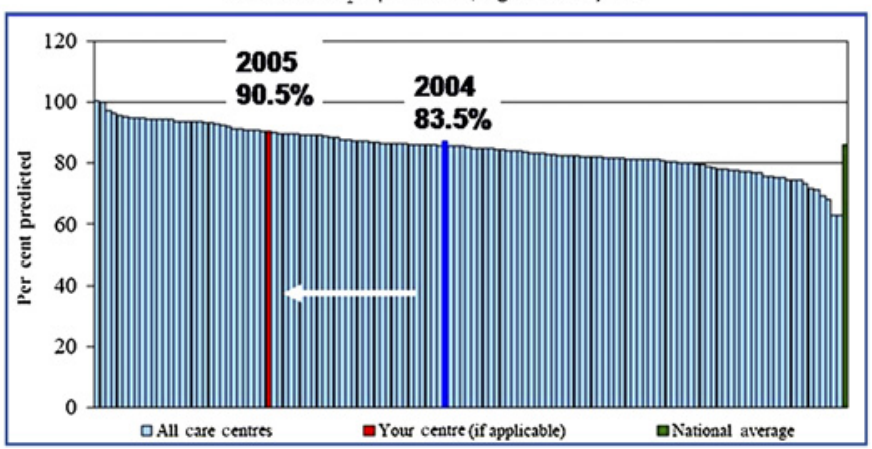

Figure 1 The two figures denote the year-to-year change in the median forced expiratory volume in one second $\left(\mathrm{FEV}_{1}\right)$ per cent predicted from 2004 to 2005 compared with other centres in the USA. The blue bar represents the Akron centres median FEV $\%$ predicted in 2004 and the red bar denotes the median $\mathrm{FEV}_{1} \%$ predicted in 2005. The green bar represents the National centre level average. The remaining bars represent all other $\mathrm{CFF}$ accredited paediatric CF centres. Reproduced with permission from Dr Nathan Kraynack. CF, cystic fibrosis; CFF, CF Foundation.

In 2003, the US CFF developed a secure internet-based portal (Port CF) to allow for the timely entry of encounterbased data and to serve as a vehicle to disseminate care guidelines and deliver patient alerts and clinical reminders at the point-of-care where it impacts on care the most. In 2006, the US CFF made centre-specific performance indicators transparent to the public and this was promoted as an initiative to 'accelerate the rate of improvement' in CF care through benchmarking. Top-performing centres are used as models of best practice with the hope of cross-pollinating successful processes to other centres, and thus reducing national variability in practice patterns and outcomes. The information gleaned from this project was disseminated in several ways including a step-by-step OI guidebook entitled 'Action Guide for Accelerating the Rate of Improvement in Cystic Fibrosis Care'. An example of some of the tools and methods employed in OI is provided in box 1 .

In addition to providing the guide, the US CFF also launched a series of action-oriented training programs entitled Learning and Leadership Collaboratives to increase the capacity for OI across the CF clinical care network. Each centre participating in one of the Learning and Leadership Collaboratives performed a OI project over the course of a year. One example noting the impact of $\mathrm{OI}$ is the change in lung function in children at the Akron Ohio CF centre after implementing a OI project to standardise the management of acute pulmonary exacerbation (figure 1).

There are several examples of how the US CFF Patient Registry has been used by CF centres to motivate OI. In 1998, the Northern New England Cystic Fibrosis Consortium (NNECFC) noticed significant variation in its screening rates for CF-related diabetes (16-78\%) and the overall rate was $17 \%$ below the national average. ${ }^{8}$ Following a 3-year OI initiative that involved feedback of data to clinicians, patient education about CF-related diabetes, and clinic system changes to trigger annual blood work and tracking of results, the NNECFC reduced variation in its centres to $71-90 \%$, and the overall rate was $4 \%$ above the national average. In another example, the Children's Memorial Hospital in Chicago was able to reduce rates of nutritional failure by documenting nutritional status at every clinic visit and targeting those patients at the highest risk for nutritional failure with a customised self-management plan. ${ }^{9}$

\section{CFF TDN: IMPROVING RESEARCH PROCESSES}

COI has not been restricted to clinical care in CF. The CFF TDN, with 77 clinical sites across the USA, has employed the same COI approach employed in the clinical arena to improve quality and efficiency in the conduct of clinical trials in CF. During the past 3 years, the US CFF has invested in the development of a web-based database system to collect study-related metrics from sites participating in CF clinical trials. Data are captured on time for IRB approval, contract approval, site activation (approval to enrolment), time to first patient enrolled and number of patients enrolled. These data have been used by the CFF TDN to initiate a benchmarking process (with funding from the US National Institutes of Health-NCRR UL1 RR025014) to identify and evaluate high performing centres based primarily on efficient study start-up and measures of enrolment performance. Given the challenges of conducting clinical research, clear pathways to successful and efficient conduct of clinical trials are needed. The results from this project will be available in the fall of 2011.

\section{OVERCOMING INERTIA: OI IMPLEMENTATION}

Although the success story of COI in CF may be considered unique as $\mathrm{CF}$ is an orphan disease affecting young people, and has been well supported by a patient foundation, there are several key lessons that can be learnt. COI in healthcare must be approached systematically with redesign of processes and strategic planning of the change process to help overcome inertia related to the status quo. The initiative requires a champion that can articulate a vision and galvanise the collective buy-in of a multidisciplinary team towards a common goal. It requires data collection and measurement to identify opportunities for improvement and to provide instant feedback following implementation of the OI initiative. Timely feedback is crucial to reinforcing good behaviour and facilitating the reiterative nature of COI. Finally, the collected data should be transparent to facilitate benchmarking and identification of best practices, which captures the true essence of COI.

Acknowledgements We would like to thank Dr Nathan Kraynack for providing figure 1 in this manuscript and Jill VanDalfsen for providing a description of the ongoing clinical research benchmarking project.

Competing interests None 
Contributors BSQ wrote a portion of this work and revised and reviewed this work. CHG wrote the other portion of this work and revised and reviewed this work.

Provenance and peer review Not commissioned; internally peer reviewed

\section{REFERENCES}

1. Closing the Quality Gap: Revisiting the State of the Science Series: Quality Improvement Interventions to Address Health Disparities. Review Protocol. Rockville, MD: Agency for Healthcare Research and Quality, 2011. http://www.ahrq.gov/clinic/ tp/gapdisptp.htm.

2. Headrick LA, Neuhauser D. Quality health care. JAMA 1994;271:1711-12.

3. Cystic Fibrosis Foundation Patient Registry. 2009 Annual Data Report. Bethesda, Maryland: Cystic Fibrosis Foundation, 2011. http://www.cff.org/research/ ClinicalResearch/PatientRegistryReport.
4. Goss CH, Mayer-Hamblett N, Kronmal RA, et al. The cystic fibrosis therapeutics development network (CF TDN): a paradigm of a clinical trials network for genetic and orphan diseases. Adv Drug Deliv Rev 2002;54:1505-28.

5. Warwick WJ, Pogue RE. Cystic fibrosis. An expanding challenge for internal medicine. JAMA 1977;238:2159-62.

6. Batalden PB, Davidoff F. What is "quality improvement" and how can it transform healthcare? Qual Saf Health Care 2007:16:2-3.

7. Improvement Methods: Getting Started. 2011. Cambridge, MA: Institute for Healthcare Improvement. http://www.ihi.org//HI/Topics/Improvement/ImprovementMethods/ (accessed 23 Jun 2011)

8. Quinton HB, O'Connor GT. Current issues in quality improvement in cystic fibrosis. Clin Chest Med 2007:28:459-72.

9. Cystic Fibrosis Center News. Chicago, IL: Children's Memorial Hospital, 2004; Volume 10. http://www.childrensmemorial.org/documents/ cfcnews winter04.pdf

\section{Pulmonary puzzle}

\section{ANSWER}

From the question on page 1103

A CT-guided percutaneous core needle biopsy of the left lower lobe cavitary nodule and a skin biopsy were performed. Pathological findings in both cases showed spindle-shaped cells with vascular channels (figure $2 \mathrm{~A}$ ) positive for human herpes virus 8 immunostaining (figure 2B). A final diagnosis of Kaposi's sarcoma (KS) was established, in the absence of positive cultures and stains for bacteria, fungi, protozoa, viruses or tumour cells from aspirated content of the cavitary lesion. The patient rapidly deteriorated and died.

Multiple pulmonary nodules with focal cavitary lesions may represent neoplasms such as bronchogenic carcinomas and lymphomas, or benign lesions including many types of infections or abscesses, immunological disorders such as Wegener granulomatosis and rheumatoid nodules, septic emboli, pulmonary infarcts, progressive massive fibrosis with pneumoconiosis, lymphocytic interstitial pneumonia, localised bronchiectasis and some congenital lesions. ${ }^{1}$

Intrathoracic KS, often in the context of AIDS, presents as nodular masses, with characteristic thickening of bronchovascular pathways, often accompanied by pleural effusions. ${ }^{2}$ Cavitation after necrosis of larger nodules is uncommon ${ }^{3}$ and should be associated with KS only after other causes, particularly infection, have been ruled out, as in this case. KS should not be thought of as exclusively associated with HIV infection. The emergence of $\mathrm{KS}$ in non-HIV persons is a rare but existent clinical condition, involving classic Mediterranean, endemic African and the iatrogenic form in patients on immunosuppressive medications ${ }^{4}$ as in this case. Corticosteroid immunosuppression can be significant even at moderate doses, as evidenced in this case and a previous report of non-HIV KS in an old person with giant cell arteritis. ${ }^{5}$

Thorax 2011;66:1108. doi:10.1136/thoraxinl-2011-200757

\section{REFERENCES}

1. Ryu JH, Swensen SJ. Cystic and cavitary lung diseases: focal and diffuse. Mayo Clin Proc 2003;78:744-52.

2. Khalil AM, Carette MF, Cadranel JL, et al. Intrathoracic Kaposi's sarcoma. CT findings. Chest 1995;108:1622-6.

3. Lai KK. Pulmonary Kaposi's sarcoma presenting as diffuse reticular nodular infiltrates with cavitary lesions. South Med J 1990;83:1096-8.

4. Hiatt KM, Nelson AM, Lichy JH, et al. Classic Kaposi Sarcoma in the United States over the last two decades: a clinicopathologic and molecular study of 438 non-HIVrelated Kaposi Sarcoma patients with comparison to HIV-related Kaposi Sarcoma. Mod Pathol 2008:21:572-82.

5. Kuttikat A, Joshi A, Saeed I, et al. Kaposi sarcoma in a patient with giant cell arteritis. Dermatol Online J 2006;12:16.
Figure 2 High-power photomicrographs of left lower lobe cavitary nodule, showing spindle-shaped cells with vascular channels $(A)$ and positive nuclear immunoreactivity for HHV-8 stain (B). HHV, human herpes virus.
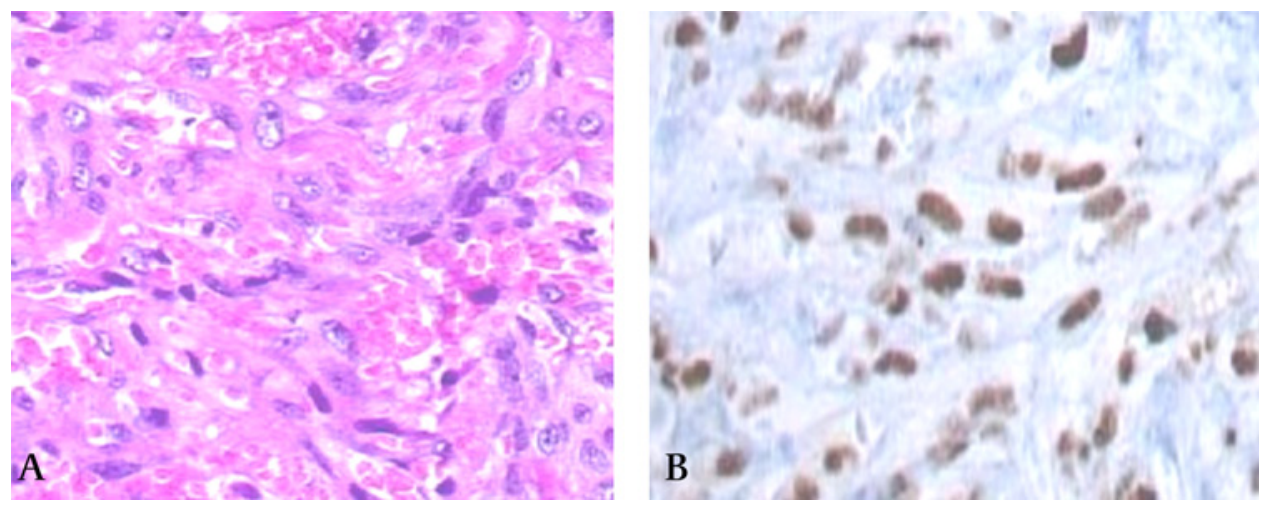